\title{
Data processing for exoplanet detection using direct imaging
}

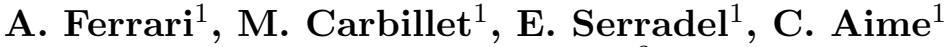 \\ and R. Soummer ${ }^{2}$ \\ ${ }^{1}$ LUAN, Universié de Nice Sophia-Antipolis, \\ 06108 Nice cedex 2, France - email: \{ferrari,marcel,serradel,aime\}@unice.fr \\ ${ }^{2}$ Department of Astrophysics, American Museum of Natural History, \\ 79th Street at Central Park West, New York, NY 10024, USA - email: soummer@amnh.org

\begin{abstract}
This communication is devoted to data processing of images obtained using an extreme adaptive optics (AO) system and a coronagraph. Specific attention is given to the following degrading factors: the residuals of atmospheric turbulence after $\mathrm{AO}$ correction and the "side" effects of the coronagraph. Relying on a statistical modeling of the measurements a test based on short exposure images is proposed. This processing, which generalizes the dark-speckle technique, takes into account the "local" variance of the complex amplitude residuals and the deterministic response of the system (i.e. without atmospheric turbulence).
\end{abstract}

Keywords. instrumentation: high angular resolution, methods: data analysis, techniques: high angular resolution, techniques: image processing, stars: planetary systems.

\section{Assumptions and data model}

Let us start considering a high-flux regime. We adopt in the sequel the model derived in Aime \& Soummer (2004). The complex amplitude resulting from the propagation of light through the atmosphere, to the telescope, and then through an adaptive optics (AO) system and a coronagraph can be decomposed into two terms in the focal plane. The first term is the deterministic response of the coronagraph, we denote it $c(\vec{x})$ where $\vec{x}$ represents the position in the focal plane. The second term comes from the atmospheric turbulence residuals not entirely corrected by the AO system and propagated through the coronagraph, $\Psi_{s}(\vec{x})$. The complex value $\Psi_{s}(\vec{x})$ is assumed to be circular Gaussian distributed: $\Psi_{s}(\vec{x}) \sim \mathcal{N}_{c}\left(0, \sigma^{2}(|\vec{x}|)\right)$. As a consequence, the instantaneous intensity $i(\vec{x})$ is written $\left|\Psi_{s}(\vec{x})+c(|\vec{x}|)\right|^{2}$. Moreover, a planet in $\vec{x}$ will basically shift $i(\vec{x})$ by a deterministic quantity $m(\vec{x})$.

The Gaussian distribution assumption for the complex amplitude is well established in a single position $\vec{x}$. However, the extension of this hypothesis to a vector containing the complex amplitudes at different positions of the focal plane is not natural, mostly due to the complexity of the transformations operated in the AO system. For this reason we will limit the study to a monodimensional statistical model. Note that, besides the fact that a multidimensional model will introduce extra unknown parameters (the spatial correlation of the complex amplitude), it leads to results which are not analytical (Ferrari et al. (2004), Tourneret et al. (2005)). To simplify the notations the position $\vec{x}$ will be omitted.

According to the previous assumptions, when $m=0 i$ is proportional to a random variable distributed as a noncentral $\chi_{2}^{2}$ distribution (Johnson et al. (1995)), also denoted 
as a Rice distribution in statistical optics. Hence, the probability distribution of $i$ is:

$$
p(i ; m, c, \sigma)=\frac{1}{\sigma^{2}} \exp \left(-\frac{i-m+c^{2}}{\sigma^{2}}\right) I_{0}\left(\frac{2 c \sqrt{i-m}}{\sigma^{2}}\right) H(i-m)
$$

where $I_{0}$ is the order 0 first type modified Bessel function. We recall the first two moments of this distribution:

$$
\mathrm{E}[i]=\sigma^{2}+c^{2}+m, \operatorname{var}[i]=\sigma^{4}+2 c^{2} \sigma^{2} .
$$

Let us now consider the low-flux regime, and in particular the processing of $M$ short exposure images. The number of detected photons in a pixel is distributed as:

$$
\operatorname{Pr}(N=n ; m, c, \sigma)=\int_{0}^{+\infty} \frac{i^{n}}{n !} \exp (-i) p(i ; m, c, \sigma) d i .
$$

When $m=0$, this writes:

$$
\operatorname{Pr}(N=n ; 0, c, \sigma)=\frac{\exp \left(-\frac{c^{2}}{\sigma^{2}}\right)}{1+\sigma^{2}}\left(\frac{\sigma^{2}}{1+\sigma^{2}}\right)^{n}{ }_{1} F_{1}\left(n+1 ; 1 ; \frac{c^{2}}{\sigma^{2}+\sigma^{4}}\right),
$$

where ${ }_{1} F_{1}(a ; b ; z)$ is a Kummer function which reduces here to a polynomial:

$$
{ }_{1} F_{1}(n+1 ; 1 ; z)=\exp (z) \sum_{k=0}^{n} \frac{n !}{k ! k !(n-k) !} z^{k} .
$$

Instead, when $m \neq 0$, Eq. (1.3) directly leads to:

$$
\operatorname{Pr}(N=n ; m, c, \sigma)=\exp (-m) \sum_{k=0}^{n} \frac{m^{n-k}}{(n-k) !} \operatorname{Pr}(N=k ; 0, c, \sigma)
$$

\section{Test derivation}

When all the parameters (here $c, \sigma$ and $m$ ) are known, the optimal test is the Neyman Pearson Detector (NPD) which consists in comparing the Likelihood Ratio (LR) of the data under both hypothesis (here $m=0$ vs. $m \neq 0$ ) to a threshold. This detector maximizes the probability detection for a fixed Probability of False Alarm (PFA), which can be tuned by the threshold value. When the parameters are unknown a suboptimal technique is the Generalized Likelihood Ratio detector where the unknown parameters are replaced by their maximum likelihood estimators under both hypothesis (see for example Bickel \& Doksum (2001)).

The estimation of $m(\vec{x})$ from $\operatorname{Pr}(N=n ; m, c, \sigma)$ is complex. However $m$ being assumed small we retain the Locally Most Powerful (LMP) test. This one relying on a Taylor expansion of the LR is optimal for $m$ close to 0 . The test decides: $m(\vec{x}) \neq 0$ if $T_{L M P}(\vec{x})>$ $\xi$ where:

$$
\begin{aligned}
T_{L M P}(\vec{x}) & =\left.\sum_{k=1}^{M} \frac{\partial \log \left(\operatorname{Pr}\left(N=n_{k} ; m, c, \sigma\right)\right)}{\partial m}\right|_{m=0} \\
& =\left.\sum_{k=0}^{\infty} \frac{\partial \log (\operatorname{Pr}(N=k ; m, c, \sigma))}{\partial m}\right|_{m=0} N_{k}=-M+\sum_{k=1}^{\infty} \beta(k ; c, \sigma) N_{k}
\end{aligned}
$$




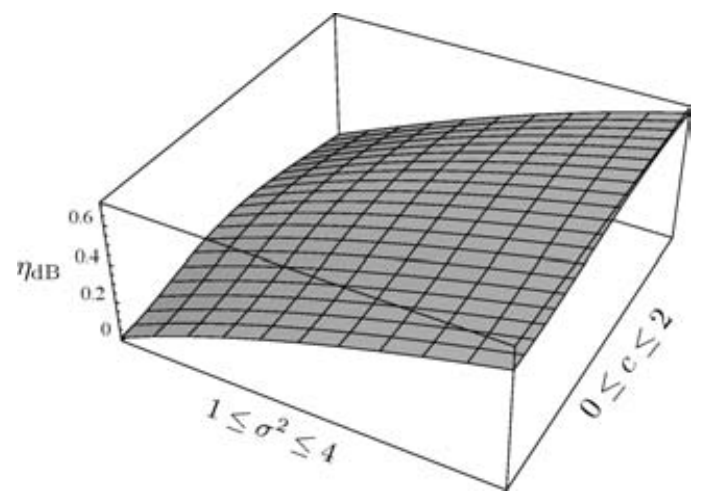

Figure 1. Comparison between the SNR of the long exposure image and the LMP test.

where $N_{k}$ is the number of times where $k$ photons are detected in $\vec{x}$ and:

$$
\beta(n ; c, \sigma)=\left(1+\frac{1}{\sigma^{2}}\right) \frac{{ }_{1} F_{1}\left(n ; 1 ; \frac{c^{2}}{\sigma^{2}+\sigma^{4}}\right)}{{ }_{1} F_{1}\left(n+1 ; 1 ; \frac{c^{2}}{\sigma^{2}+\sigma^{4}}\right)}
$$

Let us note that the well-known Dark Speckle (DS) technique, Boccaletti et al. (2000), is equivalent to the test statistic $(2.2)$ were $\forall k>0, \beta\left(k ; c, \sigma^{2}\right)=1$.

\section{Test performances}

Once the test statistic as been defined, an important point is the derivation of its distribution in order to relate the threshold to the PFA. The $N_{k}$ follows a joint multinomial distribution with index $M$ and cell probability given by Eq. (1.4) or (1.6). This distribution was used by Ferrari et al. (2005) to prove that $\mathrm{SNR}_{\mathrm{NPD}}-\mathrm{SNR}_{\mathrm{DS}}=O\left(c^{2}\right)$ for the model under scope.

However, due to the complexity of the exact distribution, we will focus on the standard asymptotic ( $M$ large) distribution of the LMP test statistic (see for example Bickel \& Doksum (2001)). When $M$ is large we have:

$$
S \stackrel{\text { def }}{=} \frac{T_{L M P}}{M I(0)} \stackrel{a}{\sim}\left\{\begin{array}{l}
H_{0}: \mathcal{N}(0,1) \\
H_{1}: \mathcal{N}(m \sqrt{M I(0)}, 1)
\end{array}\right.
$$

where $I(0)$ is the Fisher information for $m$ when $m \rightarrow 0$. A straightforward calculus shows that:

$$
I(0)=-\lim _{m \rightarrow 0} \mathrm{E}\left[\frac{\partial^{2} \operatorname{Pr}(N=n-1 ; 0, c, \sigma)}{\partial^{2} m}\right]=\sum_{n=1}^{\infty} \frac{\operatorname{Pr}(N=n-1 ; 0, c, \sigma)^{2}}{\operatorname{Pr}(N=n ; 0, c, \sigma)}-1
$$

Using (3.1) the test simplifies to:

$$
\text { decide } m=0 \text { if } S<\sqrt{M I(0)} z_{1-\mathrm{PFA}}
$$

where $z_{\alpha}$ is the $\alpha$ quantile of the standard normal distribution.

At this point, let us note that the SNR of the LMP can be compared to the SNR of the long exposure image, computed using Eqs. (1.2), thanks to the ratio:

$$
\eta=\frac{\mathrm{SNR}_{\mathrm{LMP}}}{\mathrm{SNR} \text { of long exposure image }} \propto I(0)\left(\sigma^{4}+2 c^{2} \sigma^{2}\right),
$$




\begin{tabular}{lr} 
& \\
& \\
\hline $\begin{array}{l}\text { exoplanet } \\
\text { intensity ratio wrt star } \\
\text { separation angle wrt star }\end{array}$ & $510^{-5}$ \\
\hline atmosphere+telescope & 0.5 arcsec \\
$\begin{array}{l}\text { Fried parameter } r_{0}(\text { at } 500 \mathrm{~nm}) \\
\text { number of turbulent layers }\end{array}$ & $16 \mathrm{~cm}$ \\
layers velocities & 5 \\
wave-front outer-scale $\mathcal{L}_{0}$ & $24 \mathrm{~m} / \mathrm{s}$ \\
telescope diameter & $20 \mathrm{~m}$ \\
\hline
\end{tabular}

\begin{tabular}{lr}
\hline AO system & \\
sensor type & Shack-Hartmann \\
sensor configuration & $40 \times 40$ \\
number of modes & 744 \\
time-filter type & pure integration \\
\hline coronagraph & Lyot \\
coronagraph type & $2 \lambda / D$ \\
mask diameter & $0.78 D$ \\
Lyot stop diameter & \\
\hline imaging device & $(2.2 \mu \mathrm{m})$ \\
wavelength band & $\approx 1$ \\
nb. of planet phot./ms & $1 \mathrm{~ms}$ \\
short exposure & $5 \mathrm{~ms}$ \\
decorellation interval & $800 \mathrm{~ms}, M=800$ \\
long exposure & \\
\hline
\end{tabular}

Table 1. Main parameters of the data simulation.

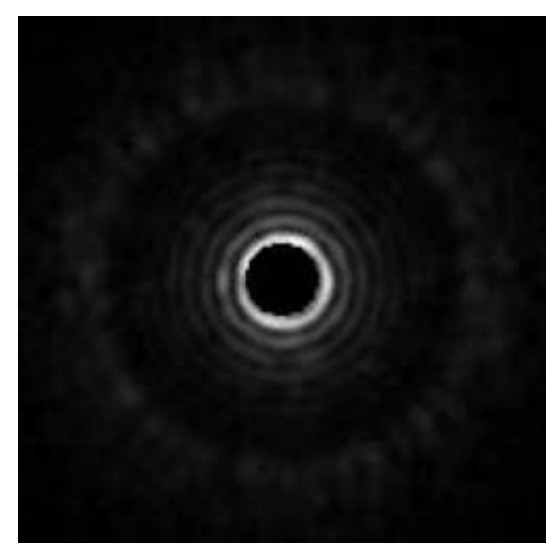

Figure 2. Long exposure masked image.
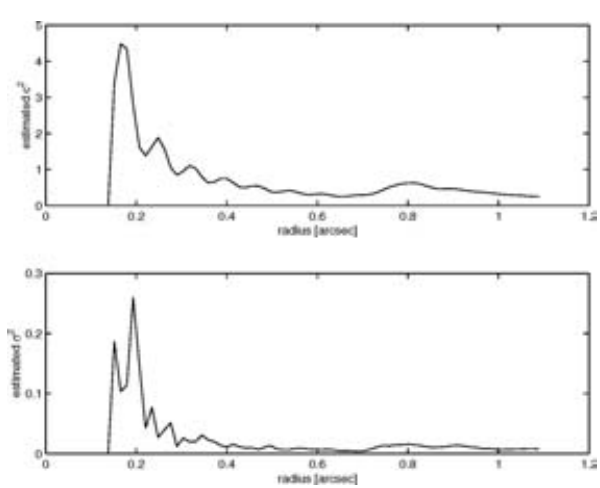

Figure 3. Estimated $c^{2}(|\vec{x}|)$ and $\sigma^{2}(|\vec{x}|)$.

A plot of $\eta$ as a function of $c$ and $\sigma^{2}$ is given in Fig. 1. It shows that the benefit of the proposed detection procedure increases principally with the variance of the turbulence residual. Note that this gain is relevant only in the photon noise limited case, in particular the read-out noise is not considered.

\section{Simulations}

Table 1 contains the main physical parameters of the performed numerical simulation, made with the Software Package CAOS (Carbillet et al. (2005)), while Fig. 2 shows the resulting long-exposure image, obtained integrating the data cube short exposures.

Computation of the test statistic $S(\vec{x})$ requires the knowledge of $c^{2}(|\vec{x}|)$ and $\sigma^{2}(|\vec{x}|)$. The first could be obtained by a calibration procedure. Analytical expressions of the second have been recently proposed by Fusco \& Conan (2004) and Chelli (2005). However, the solution retained here was to estimate $c^{2}(|\vec{x}|)$ and $\sigma^{2}(|\vec{x}|)$ directly from the data. These quantities are computed solving Eq. (1.2) where the first and second order moments of $i(\vec{x})$ are estimated by sample averaging on rings of radius $|\vec{x}|$. 


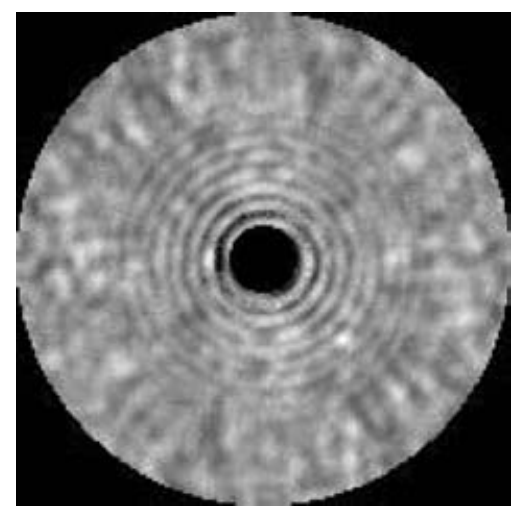

Figure 4. Test statistic $S(\vec{x})$ of the LMP test.

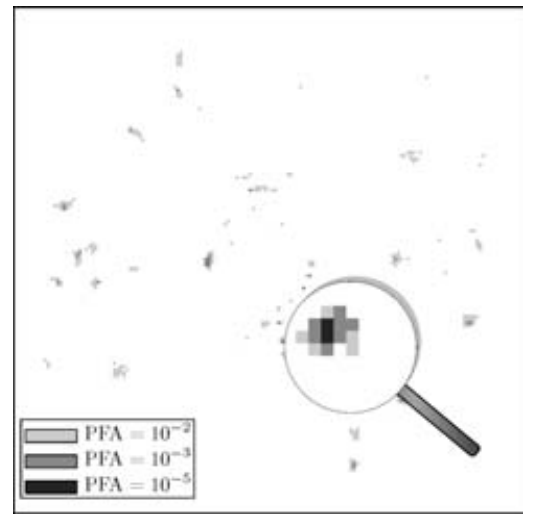

Figure 5. Result of the LMP test.

The result is given in Fig. 3. Figure 4 shows the test statistic $S(\vec{x})$ and Fig. 5 the result of the test for different values of the PFA. The lens is at the location of the simulated planet. In this simulation the planet is the unique event detected with the lower PFA.

\section{Conclusions}

We have derived a test based on short exposure images for the direct detection of faint unresolved objects in post-AO imaging data. A detailed simulation of the atmospheric and instrumental effects (8-m class telescope, $40 \times 40$ elements AO, Lyot coronagraph, K-band imaging) was performed. Our test was applied to a faint companion $\left(510^{-5}\right.$ relative intensity) at 0.5 arcsec from the main bright star, leading to promising results. Further developments include modeling of additional noise contributions and estimation procedure of the variance of the $\mathrm{AO}$ residual.

\section{References}

Aime, C. \& Soummer, R. 2004, EUSIPCO (Vienna)

Bickel, P.J. \& Doksum, K.A. 2001, Mathematical Statistics (Prentice Hall)

Boccaletti, A., Moutou, C., \& Abe, L. 2000, Astron. Astrophys. Suppl. Ser., 141, 157

Carbillet, M., Vérinaud, C., Femenía, B., et al. 2005, MNRAS 356 (4), 1263

Chelli, A. 2005, Astron. Astrophys. 441 (3), 1205

Ferrari, A., Letac, G., \& Tourneret, J.-Y. 2004, EUSIPCO (Vienna)

Ferrari, A., Tourneret, J.-Y., Serradel, E., \& Aime, C. 2005, GRETSI (Louvain-la-Neuve)

Fusco, T. \& Conan, J.-M. 2004, J. Opt. Soc. Am. A, 21 (7), 1277

Tourneret, J.-Y., Ferrari, A., \& Letac, G. 2005, Statistical Signal Processing Conference (Bordeaux)

Johnson, N. L., Kotz, S., \& Balakrishnan, N. 1995, Continuous Univariate Distributions, Vol. 2 (John Wiley) 

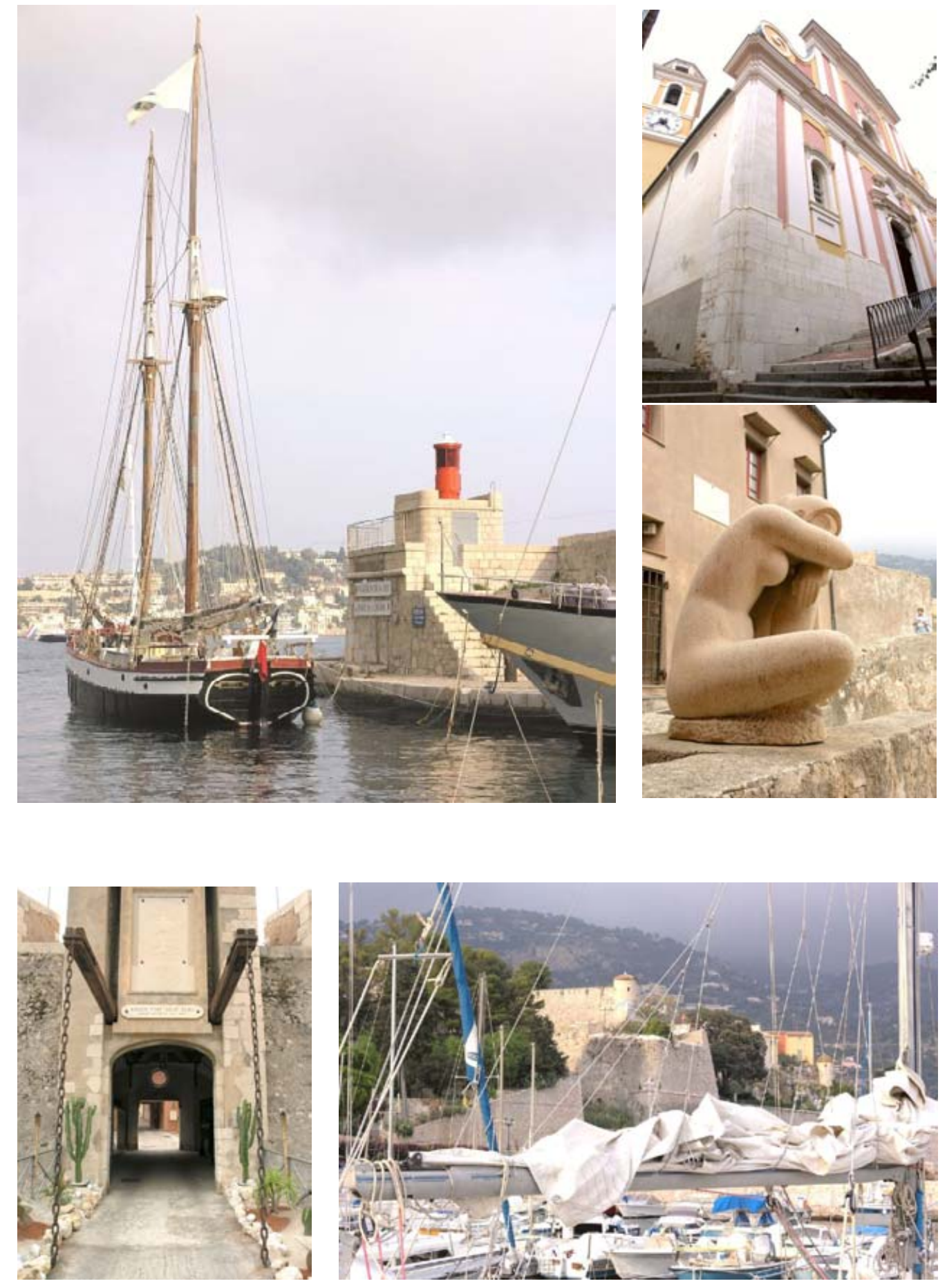

Photographs (this page only): Thomas Aime. 